\title{
Kernos
}

Revue internationale et pluridisciplinaire de religion grecque antique

12 | 1999

Varia

\section{N. Chr. STAMPolidis, Anti Poina. "Reprisals"}

\section{Eleni Georgoulaki}

\section{(2) OpenEdition}

\section{Journals}

Édition électronique

URL : http://journals.openedition.org/kernos/730

DOI : $10.4000 /$ kernos.730

ISSN : 2034-7871

Éditeur

Centre international d'étude de la religion grecque antique

Édition imprimée

Date de publication : 1 janvier 1999

Pagination : 303-305

ISSN : 0776-3824

Référence électronique

Eleni Georgoulaki, « N. Chr. stampolidis, Anti Poina. "Reprisals" », Kernos [En ligne], 12 | 1999, mis en ligne le 13 avril 2011, consulté le 10 mars 2021. URL : http://journals.openedition.org/kernos/730 ; DOI : https://doi.org/10.4000/kernos.730 
ciennes images, comme les animaux, les doubles cornes, les doubles haches, non plus comme symboles indépendants mais comme attributs.

Pour la Grèce, c'est Mary Voyatzis qui pose la question d'un éventuel héritage assumé par les déesses grecques des périodes archaïque et classique (From Athena to Zeus. An A-Z Guide to the Origins of Greek Goddesses). Parfaitement consciente du caractère périlleux d'une réflexion globale et théorique sur le sujet, elle propose l'analyse précise du site d'un sanctuaire particulier, celui d'Athéna Aléa à Tégée où elle a fouillé en collaboration avec les Norvégiens. La présentation du matériel mis au jour et son interprétation mesurée permettent de dessiner l'image d'une déesse dont les intérêts diversifiés ont évolué au fil du temps, en s'adaptant aux besoins des fidèles. Aléa pourrait être l'ancien nom de la déesse en question. Son association à Athéna ne semble pas antérieure à la fin du $\mathrm{VII}^{\mathrm{e}}$ ou au début du $\mathrm{VI}^{\mathrm{e}}$ siècle. Elle propose ensuite un bref panorama des autres divinités d'Arcadie, essentiellement fondé sur l'ouvrage de M. Jost, Sanctuaires et cultes d'Arcadie (Paris, 1985), puis elle élargit le cadre à d'autres régions de Grèce, comme pour justifier le titre de l'article, « un guide de A à $Z$ ». Le propos se fait alors plus englobant : la chute des palais mycéniens a entraîné en certains lieux l'arrêt manifeste des activités cultuelles, qui se sont pourtant poursuivies dans d'autres. L'isolement des communautés entre le $\mathrm{xI}^{\mathrm{e}}$ et le $\mathrm{Ix}^{\mathrm{e}}$ siècle a entraîné des variations dans le développement religieux à l'échelon local, encore accrues par l'arrivée de nouveaux venus, porteurs d'autres conceptions et pratiques religieuses. La conclusion de l'étude recueille les fruits de cette approche mesurée et prudente : au début de l'époque archaïque, beaucoup de divinités sont honorées dans les grands sanctuaires, mais ceux des déesses sont incontestablement plus nombreux et plus importants. Apollon est le seul dieu masculin à jouer un rôle essentiel à l'époque. Il est concevable que la prédominance des déesses et les traits communs qu'elles partagent soient un héritage de la période mycénienne.

Cet ouvrage est clair, bien documenté, et il offre une vision équilibrée d'une problématique qui a longtemps empoisonné l'histoire des religions en général, et de la religion grecque en particulier. En témoigne encore un ouvrage récent dédié à la mémoire de Maria Gimbutas et au titre significatif : Die Madonna und ibre griecbiscben Töchter ${ }^{1}$.

Vinciane Pirenne-Delforge (FNRS - Université de Liège)

Nicolas Chr. Stampolidis, Anti Poina. "Reprisals". Contribution to the study of customs of the geometric-archaic period, Rethymno, University of Crete, 1996. 1 vol. $23 \times 29 \mathrm{~cm}, 253$ p., 224 fig. 2 pl. (Eleutherna. Sector III, 3).

Vu que la fouille de la nécropole d'Eleutherna, en Crète occidentale, est toujours en cours, on comprend l'embarras des chercheurs désireux d'interpréter les vestiges anciens découverts là-bas. En effet, les suggestions proposées risquent d'être bouleversées par des découvertes à venir.

Pourtant, dans cette étude, l'A. entreprend la tâche difficile de discuter un charnier de fouille, dont la mise au jour n'a pas été complétée. Dans l'introduction de son livre [p. 15], il souligne que son but est de mettre en évidence une découverte particulière, qui risquerait de passer inaperçue jusqu'à la publication

1 Harald HaArmann, Die Madonna und ibre griecbiscben Töchter. Rekontruktion einer kulturbistoriscben Genealogie, Hildesheim, Zürich, New York, Georg Olms Verlag, 1996 (compte rendu à paraître dans L'Antiquité Classique, 68 [1999]). 
définitive du site, et de proposer une interprétation qui cerne au plus près la réalité des données disponibles.

Dans ce cadre, l'A. essaie de discerner le rôle particulier que le sens des représailles a pu jouer au sein du rituel funéraire aux époques archaïque et géométrique. Pour parvenir à tisser cette image globale, il rassemble des données aussi bien archéologiques que littéraires.

La première partie de l'ouvrage comprend un développement détaillé de la fouille du cimetière, correspondant aux crémations, et du matériel archéologique provenant de celles-ci. La fouille poursuivie sur les flancs d'une colline a révélé l'existence de deux feux funéraires successifs, en activité pendant plus de 30 ans (720-690 av. J.-C.) [p. 25 sq.]. Pendant l'été 1991, une trouvaille exceptionnelle a été mise au jour : dans le «pyra $A$ », on repéra les restes d'un squelette sans tête. Le pelvis du squelette était placé sur une brique, son épine dorsale étant trop courbée. Il est remarquable que les restes des bras du squelette n'aient pas été trouvés devant ou à côté, mais bien au-dessous du corps. En plus, une hache et un fragment d'un couteau en fer ont été mis au jour au-dessous du cou [p. 32-35].

Ensuite, l'examen chronologique et typologique des objets en métal et en argile, provenant du site, est menée avec soin [p. 44-69]. Chaque objet y est décrit soigneusement et accompagné de sa bibliographie.

Le chapitre suivant de l'exposé est consacré à l'examen scientifique des restes humains, provenant de la nécropole. Le bûcher funéraire A comprenait deux urnes funéraires, contenant à leur tour les restes de trois personnes : un mâle d'âge adulte, qui a probablement subi une mise à mort violente, une femme d'âge moyen et les ossements d'un troisième mort. Les ossements du dernier ont été brûlés plutôt que calcinés. D'après l'anthropologue, ils appartiennent à un individu mort probablement à la suite d'une décapitation [p. 70-77].

Dans le chapitre suivant, l'A. essaie d'interpréter les vestiges archéologiques découverts dans le «pyra A ». Il souligne le fait que les deux morts principaux ont été calcinés et étendus sur le dos. L'homme étant accompagné de ses armes, on peut supposer qu'il s'agissait d'un soldat. La femme était probablement sa compagne. Au contraire, le squelette sans tête n'a pas été incinéré et ne semble pas avoir bénéficié du même traitement que les deux autres corps [p. 78-91]. Dans ce chapitre, on peut regretter un certain nombre de répétitions et de citations des pages antérieures du livre. Cette légère incohérence est signalée dans la lettre critique envoyée par des anthropologues et publiée à la fin du volume [p. 204].

Le témoignage des sources écrites méritait bien quelques pages. En effet, elles permettent de combler les lacunes de notre connaissance par le biais de l'approche comparative. Ainsi, la deuxième partie de l'ouvrage reprend les éléments relatifs aux poèmes épiques. De la comparaison du matériel archéologique avec Homère [p. 93-148], l'A. tire quantité de remarques dont il faut ici limiter le relevé à un ou deux échantillons. En ce qui concerne l'exécution des douze prisonniers de guerre sur la tombe de Patrocle, l'A. croit que deux faits auraient provoqué la vengeance des parents de Patrocle vis-à-vis des prisonniers : la tension émotionnelle de leurs parents et le fait que Patrocle appartenait à un rang élevé de la société [p. 103].

Dans la troisième partie de l'ouvrage, l'A. remet les données d'Eleutherna dans leur contexte chronologique et historique [p. 156-189]. Un intérêt particulier pour la loi de Dracon et les institutions de Crète en même temps que le développement des cités grecques sont au cœur du propos [p. 179-189]. En effet, l'A. cherche à voir dans les peines censées avoir été infligées aux criminels le résultat d'une structure sociale : à l'époque des tyrans, l'exécution d'un criminel sur la tombe de sa victime serait devenue institutionnelle. Dans ce cadre, l'A. croit que 
le soldat mort à Eleutherna serait un défunt de haut rang, et aurait bénéficié d'un rituel organisé. Il aurait probablement été tué par l'homme décapité, un personnage de rang inférieur. Le dernier aurait été condamné à être décapité sur la tombe de sa victime et mutilé en ses extrémités [p. 185-189]. Le dernier chapitre de l'exposé reprend la bibliographie sur les croyances grecques relatives à l'âme.

En conclusion, l'A. propose une hypothèse qui semble concilier les données archéologiques. Il s'agit de faire de la tombe d'une personne de haut rang le lieu d'exécution d'une autre personne [p. 201]. Cette tentative d'interprétation paraît plausible, mais on pourrait émettre quelques réserves sur les détails : en effet, l'ensemble des indications de la fouille offre une image plutôt floue de la fonction du site comme lieu de représailles. Dans l'état actuel de nos connaissances, il est difficile de voir dans le squelette sans tête d'Eleutherna le résultat d'une structure sociale particulière ou d'envisager l'existence généralisée d'un traitement des prisonniers de guerre. En l'absence de solution immédiate et d'éléments de comparaison archéologiques, il faudrait sans doute se limiter à des hypothèses plus prudentes.

La variêté et la qualité des illustrations du livre sont remarquables. La consultation des illustrations est facilitée par une liste des figures. L'ouvrage est complété par la bibliographie et un index.

Eleni Georgoulaki (Athènes)

Robin HägG (éd.), The Role of Religion in the Early Greek Polis. Proceedings of the Third International Seminar of Ancient Greek Cult, organized by the Swedish Institute at Athens, 16-18 October 1992, Stockholm, Åström, 1996. 1 vol. $16,5 \times 24,2 \mathrm{~cm}, 176$ p. , ill. (Acta Instituti Atbeniensis Regni Sueciae, Series in-8 $\left.{ }^{\circ}, 14\right)$. ISBN : 91-7916-033-6.

Contrairement aux deux volumes précédents, qui étaient axés, l'un sur l'iconographie ${ }^{1}$, l'autre sur l'épigraphie ${ }^{2}$, cette publication du troisième séminaire international sur les cultes anciens de la Grèce est pluridisciplinaire, faisant intervenir l'archéologie, l'iconographie, la littérature et l'épigraphie. L'objet est d'étudier le rôle de la religion lors de la formation et des débuts de l'histoire de la polis. Les contributions abordent des sujets très variés, dont on aura ici un aperçu. - Irad Malkin ( "The polis between myths of land and territory », p. 9-19) s'intéresse à l'interférence du mythe et de la politique dans la polis primitive. L'essentiel de l'article porte sur la légende libyenne de la victoire d'Héraklès sur Antée : elle reflète l'ouverture du pays à la civilisation et ne cesse, pendant deux siècles, de s'étendre vers l'Est, selon les acquisitions territoriales et les fondations coloniales, au fur et à mesure de l'extension du territoire de Cyrène. - Walter Burkert ("Greek temples-builders: who, where and why? ", p. 21-29) souligne le fait que l'édification d'un temple est d'abord une démonstration de prestige, en forme de remerciement aux dieux (le butin de guerre doit avoir joué dès les premiers temps un rôle important dans le financement des monuments); il insiste ensuite sur deux motifs religieux: la compensation d'un acte impie et la réponse à un oracle ou à des signes divins. Au total, le hasard, prérogative des dieux, et le choix des hommes se conjuguent pour créer le paysage religieux d'une cité. -

1 R. HägG (éd.), The iconograpby of Greek cult in the Archaic and Classical periods, Liège-Athènes, 1992 (Kemos, suppl. 1).

2 R. Ḧ̈GG (éd.), Ancient Greek cult practice from the epigrapbical evidence, Stockholm, 1994 (ActaAtb-8, 13 ). 